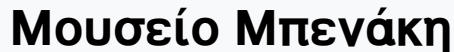

Tóp. 4 (2004)

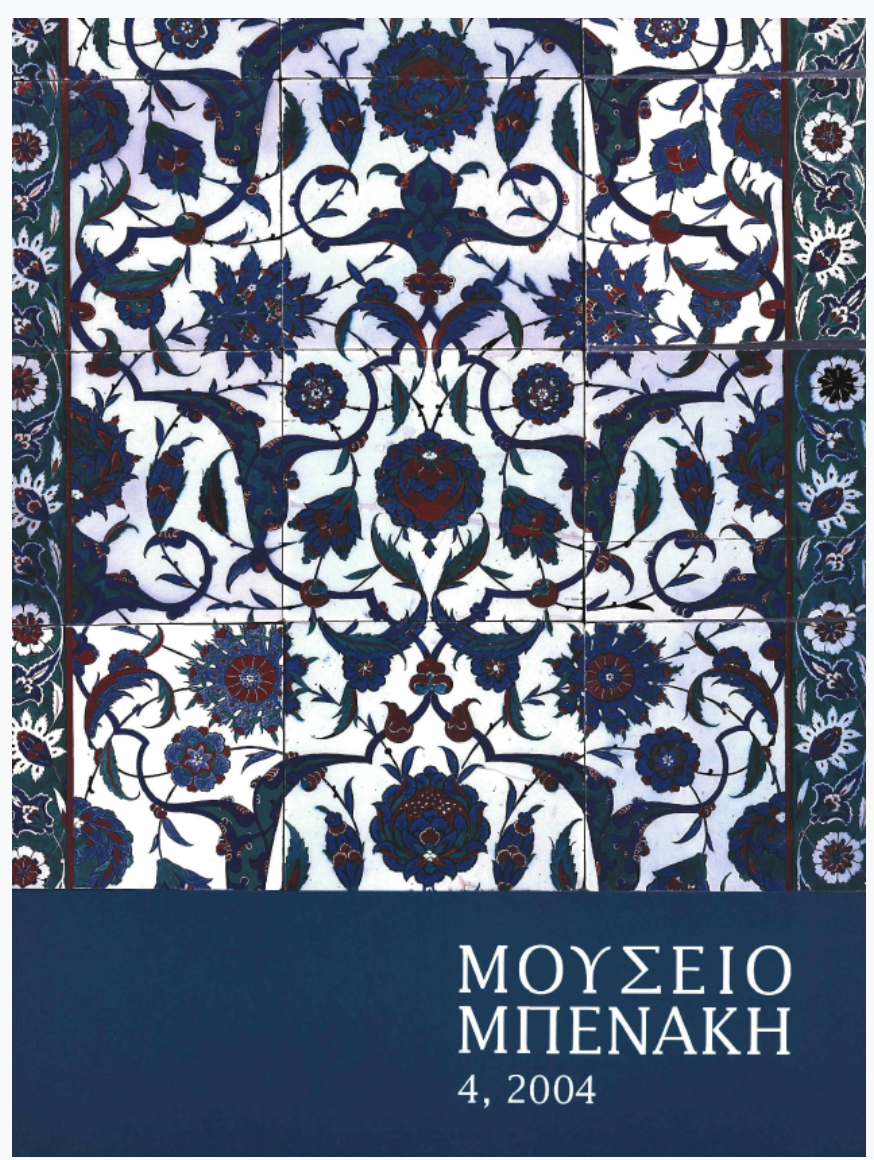

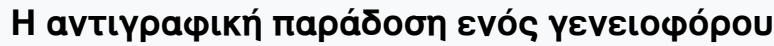
$\varepsilon \lambda \lambda \eta$ viкоú портраítou

Emmanuel Voutiras

doi: $\underline{10.12681 / \text { benaki.18248 }}$

Copyright @ 2018, Emmanuel Voutiras

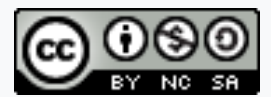

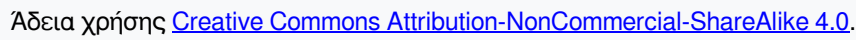

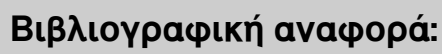

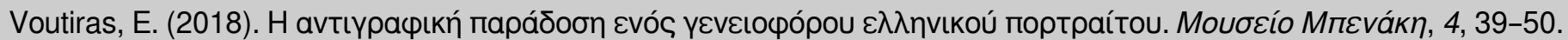
https://doi.org/10.12681/benaki.18248 


\section{Zur Überlieferung eines bärtigen griechischen Porträts}

DIE BEREITS ansehnliche Antikensammlung des Benaki Museums in Athen ist vor kurzem durch eine Schenkung um ein interessantes Stück reicher geworden (s. Nachtrag). Es handelt sich um einen qualitätvollen, leicht überlebensgroßen Marmorkopf, der nach Angabe der früheren Besitzerin ein altes Erbstück ist und aller Wahrscheinlichkeit nach aus Athen stammt.' Dargestellt ist ein älterer Mann mit auffällig langem Bart und wirrem Haupthaar (Abb. 1-4). Das Material ist weißer feikörniger, wohl pentelischer Marmor. ${ }^{2}$ Gebrochen ist die Nase, der größte Teil der rechten Augenbraue und die rechte Seite des Schnurrbarts. Außerdem sind vielenorts größere oder kleinere Absplitterungen zu bemerken. Die gesamte Oberfläche ist stark abgerieben, wobei vor allem die Haar- und die Bartlocken gelitten haben. Der Erhaltungszustand verdient aber eine weitere Kommentierung; denn auf der rechten Seite des Kopfes ist ein großer Teil der Oberfläche, welcher das Haupthaar sowie einen Teil des Halses und des Bartes umfaßt, völlig abgesplittert. Diese Zerstörung setzt sich im Oberund Hinterkopf fort. Am schlimmsten betroffen ist das Hauphaar vorne rechts, von der Stirnmitte bis zur Schläfe, wo eine ziemlich dicke Marmorschicht abgesprungen ist. Diese Art von Beschädigung kann nicht durch Verwitterung entstehen. Auch die Abreibung und die Einwirkung von Wasser können wegen der rauhen Bruchfläche als Ursache ausgeschlossen werden. Wahrscheinlich ist der Zustand der rechten Seite des Kopfes als Folge starker Hitzeeinwirkung zu erklären, durch die die Oberfläche des Marmors zersplittert ist und z.T. größere Stücke abgefallen sind. ${ }^{3}$ Der Kopf dürfte also bei einem
Brand stark in Mitleidenschaft gezogen worden sein, wohl noch bevor er unter die Erde kam. Die Unterseite bildet eine schräge, leicht konvexe tellerartige Fläche, die unter dem Bart kräftiger gewölbt ist. Die Form dieser sorgfältig gepickten Fläche läßt darauf schließen, $\mathrm{da}$ der Kopf ursprünglich in eine Herme eingelassen war, wobei er in das entspechend abgeschrägte, leicht konkave obere Ende des Hermenschaftes eingesetzt wurde. ${ }^{4}$ Hinten sind am unteren Rande des Nackens zwei grobe, ungefähr dreieckige Vertiefungen sichtbar, die die antike Patina durchbrechen und deshalb neuzeitlich sein müssen; eine weitere derartige Vertiefung befindet sich auf der Unterseite des Kopfes. Es bleibt ungewiß, ob die leichte Erhebung am linken Halsrand als Ansatz einer Mantelfalte zu deuten ist, die sich auf der Herme fortgesetzt haben dürfte. Auf jeden Fall wies der Kopf eine deutliche Wendung nach links auf, wie vor allem die Rückansicht (Abb. 4) zeigt (s. unten).

Was die Datierung des neuen Porträts im BenakiMuseum betrifft, so ist vor allem die Gestaltung der Augenpartie aufschlußreich. Die relativ tiefliegenden schmalen, mandelförmigen Augen werden von gleichmäßig dünnen, kantigen Lidern umrahmt. Die Iris ist durch Einritzung und die Pupille durch eine bohnenförmige Eintiefung wiedergegeben. Diese Merkmale sind charakteristisch für attische Porträtköpfe der mittleren Kaiserzeit, wobei die nächsten Parallelen der Regierungszeit des Antoninus Pius angehören. Sehr ähnlich ist in dieser Hinsicht ein qualitätvoller, überlebensgroßer, bärtiger Kopf von der Athener Agora, der in die frühantoninische Periode zu datieren ist. ${ }^{5}$ Dieser Bildniskopf steht 

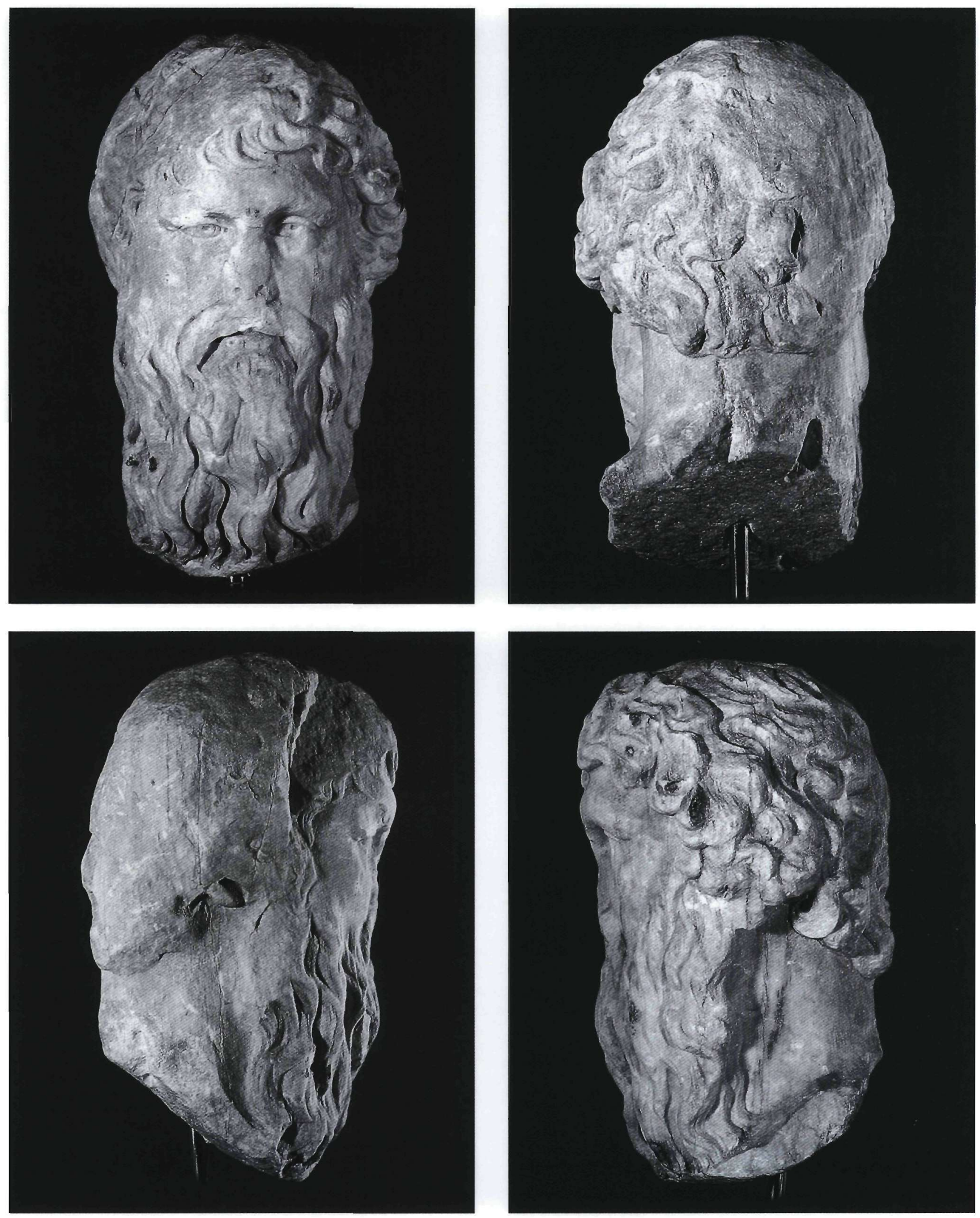

Abb. 1-4. Bärtiger Marmorkopf. Athen, Benaki Museum, Inv. Nr. 39282 (Fotos: S. Skopelitis). 

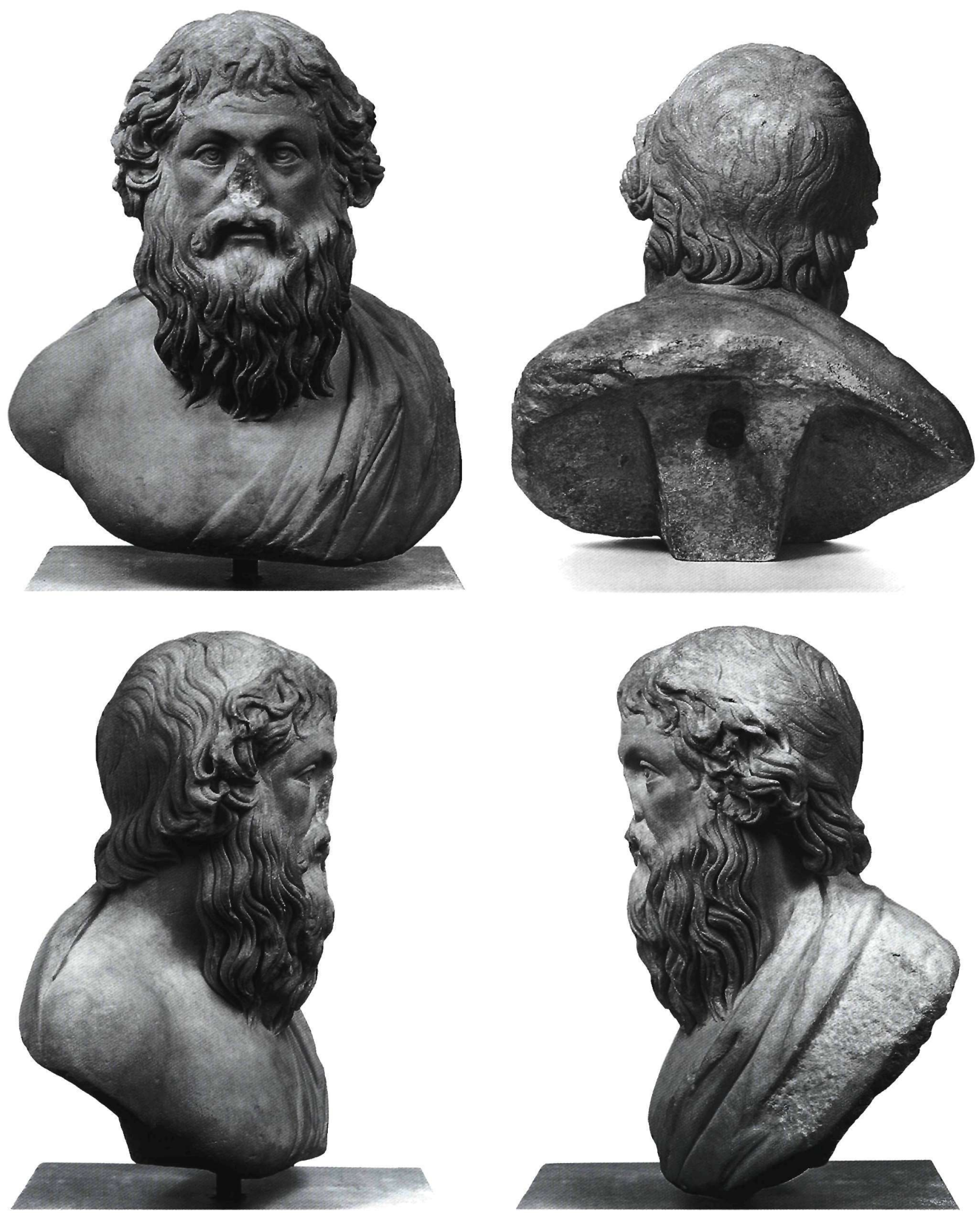

Abb. 5-8. Marmorbüste eines bärtigen Mannes. Malibu, J. Paul Getty Museum, Inv. Nr. 71.AA.284 (Fotos: Museum). 

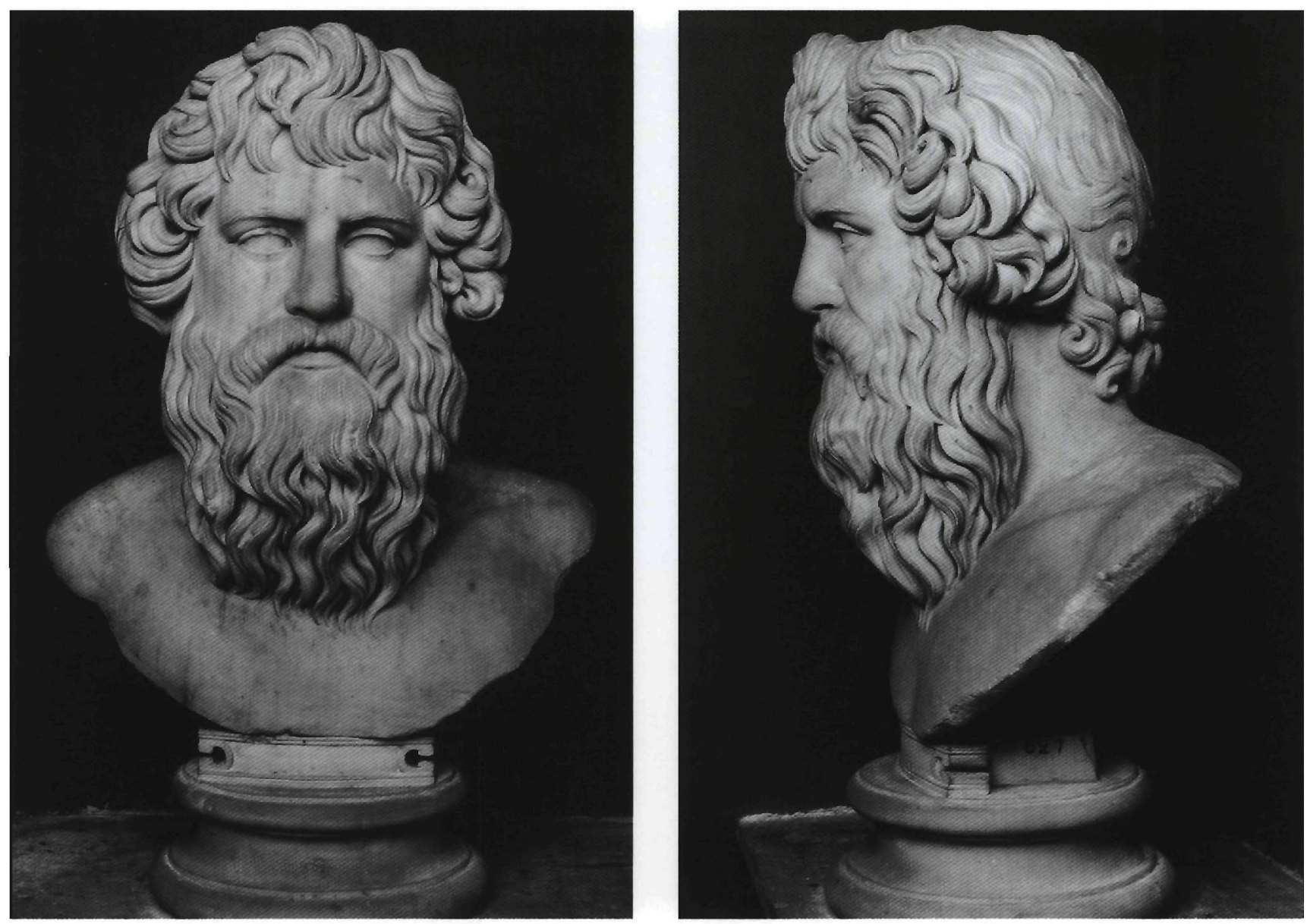

Abb. 9-10. Marmorbüste eines bärtigen Mannes. Vatikan, Museo Pio Clementino, Inv. Nr. 627 (Fotos: DAI Rom).

dem im Benaki-Museum auch hinsichtlich der weichen Modellierung des Inkarnats sowie der Ausführung der Haar- und Bartlocken ${ }^{6}$ so auffallend nahe, daß es gerechtfertigt erscheint, die beiden Werke derselben Bildhauerwerkstatt zuzuschreiben. ${ }^{7}$ Unser Kopf ist also aller Wahrscheinlichkeit nach etwa um die Mitte des 2. Jhs. n. Chr. in Athen entstanden.

$\mathrm{Daß}$ der Kopf im Benaki-Museum keine originale Schöpfung der Kaiserzeit ist, sondern vielmehr auf ein griechisches Original zurückgeht, liegt schon aufgrund seiner typologischen Merkmale nahe, wobei dem imponierenden, langlockigen Bart eine besondere Bedeutung zukommt. ${ }^{8}$ Bei näherem Hinsehen erweist sich das Stück in der Tat als Replik eines eindrucksvollen, wenngleich namenlosen Porträttypus, von dem zwei weitere Kopien in Büstenform bekannt sind, eine im J. Paul Getty Museum in Malibu (Kalifornien)' (Abb. 5-8) und eine im Vatikan ${ }^{10}$
(Abb. 9, 10). Was die Herkunft der ersten betrifft, so weiß man nur, daß sie aus dem New Yorker Kunsthandel erworben wurde, wohin sie über den römischen Kunsthandel gelangte. ${ }^{11}$ Vermutlich stammt diese Büste aus Italien. Über den Fundort der zweiten Replik liegen dagegen genaue Informationen vor. Diese wurde nämlich 1769 von dem schottischen Künstler Gavin Hamilton in der Villa Hadriana bei Tivoli gefunden und kurz darauf dem Vatikan zum Kauf angeboten. ${ }^{12}$

Die neue Replik ist für die Rekonstruktion des Originals deshalb besonders wichtig, weil sie als einzige nicht durch Überarbeitung entstellt ist. Was das Büstenbildnis in Malibu betrifft, so stellte bereits J. Raeder fest, daß das gesamte Gesicht samt Mundpartie und Schnurrbart umgestaltet worden ist. ${ }^{13}$ Ein roher Steg, der rechts und links das Schläfenhaar begleitet (am deutlichsten in den Seitenaufnahmen, Abb. 6, 7), markiert den Übergang zur 
tiefer liegenden Schicht des umgearbeiteten Gesichts und zeigt den Umfang des späteren Eingriffs, der hier nicht erneut im Einzelnen beschrieben werden muß. Den Beobachtungen Raeders ist nur hinzuzufügen, daß auch die Haarlocken auf der Stirn und dem Oberkopf von der Überarbeitung betroffen sind, wie die rauhe Oberfläche nahelegt und ein Vergleich mit der entsprechenden Partie der Replik Benaki bestätigt. Dabei lassen die verbliebenen Spuren eindeutig erkennen, daß die Stirnlocken der Replik Getty ursprünglich in Form und Verlauf denen der Replik Benaki genau entsprachen. Die Mantelfalten sind, wie Raeder beobachtet hat, wohl gleichzeitig mit dem Gesicht überarbeitet worden. Die Umgestaltung der Replik Getty wurde von Raeder aus stilistischen Gründen in das frühe 5. Jh. n. Chr. datiert, was durchaus plausibel erscheint. ${ }^{14}$ Der lange Bart wäre zwar bei einem Porträt dieser Zeit ungewöhnlich, es ist aber denkbar, daß der Dargestellte als christliche Figur umgedeutet wurde. ${ }^{15}$ Unklar bleibt, ob die grobe Beschneidung des linken Büstenandes, wo zusätzlich ein Streifen vom Mantel roh abgehauen wurde (Abb. 6), ${ }^{16}$ und die Entfernung des zugehörigen Sockels mit der erschlossenen Wiedereverwendung zusammenhängen oder von einem weiteren, noch späteren Eingriff zeugen. ${ }^{17}$

Neuzeitliche Überarbeitung zeigt andererseits die Replik im Vatikan. Der unrelgelmäßige Verlauf und der geringe Umfang des Büstenausschnitts lassen den Schluß zu, daß die ursprünglich wohl erheblich größere Büste neu beschnitten wurde -vermutlich wegen einer Beschädigung des Randes und um sie zugleich für den Transport leichter zu machen. ${ }^{18}$ Raeder bemerkt weiter, $\mathrm{daß}$ "an der Oberfläche die Verwitterungsspuren beseitigt" wurden. ${ }^{12}$ In der Tat gingen aber die Eingriffe des Restaurators an einigen Stellen ziemlich tief. So ist auf der linken Schulter die ursprüngliche Oberfläche völlig abgearbeitet worden. Von größerer Bedeutung ist aber die Überarbeitung der Stirnlocken, die deutlich flacher und kantiger als die umliegenden Haarlocken gearbeitet sind. Ein Vergleich mit der entsprechenden Partie der Replik Benaki zeigt, daß diese Locken ursprünglich länger und dicker gewesen sein müssen. Dasselbe gilt für das untere Ende der Bartlocken. Auch hier hat der moderne Restaurator eine relativ dicke Marmorschicht abgetragen, wie die sowohl auf der Vorder- als auch auf der Seitenansicht (Abb. 9, 10) sichtbare ursprüngliche Umrißlinie der auf die Brust fallenden Lockenspitzen eindeutig zeigt. Auf der linken Seite des Bartes, dort wo die kurzen Locken des Schnurrbarts enden (Abb. 9), ist wohl, wie der Vergleich mit der entsprechenden Partie der Replik Benaki zeigt (Abb. 1), eine lange Bartsträhne weggemeißelt und die Stelle entsprechend überarbeitet worden. Die beschriebenen neuzeitlichen Eingriffe bieten eine Erklärung für die Abweichung der Vatikanischen von den beiden anderen Repliken in Bezug auf die Form der Stirn- und Bartlocken. Ob schließlich die zwei nach hinten gebogenen, auf dem Hals liegenden, dünnen Bartsträhnen an der linken Seite der Replik Benaki (Abb. 2) ursprünglich auch bei der Vatikanischen vorhanden waren und von dem Restaurator entfernt wurden damit ein sauberer Kontur entsteht, muß mangels Autopsie offen bleiben. Wahrscheinlich wurde die Restaurierung der Vatikanischen Büste von ihrem Entdecker veranlaßt, wohl in der Absicht, den Wert der zu verkaufenden Skulptur zu steigern, die er bezeichnenderweise als "singular for its high preservation" anpries. ${ }^{20}$

Die drei erhaltenen Repliken unseres Porträts stimmen -soweit sie nicht überarbeitet sind- sowohl in ihren allgemeinen Zügen als auch in den motivischen Einzelheiten miteinander überein. ${ }^{21}$ Allerdings übertrifft der Kopf im Benaki Museum, trotz seines schlechten Erhaltungzustandes, die beiden anderen in der Güte der Ausführung. Auch dürfte er dem Original am nächsten stehen, nicht nur weil er von späteren Eingriffen unberührt geblieben ist, sondern vor allem, weil er im Gesamteindruck und im Detail die vom Zeitstil des Kopisten am wenigsten beeinflußte lectio difficilior bietet. $^{22}$ Die Vatikanische Büste ist im Vergleich flach und trocken modelliert, ganz im Sinne des hadrianischen Klassizismus; ${ }^{23}$ auch dürfte sie aufgrund ihrer auffälliger Größe das Original vergrößert wiedergeben (s. unten). Trotzdem ist diese Replik, wie Raeder mit Recht hervorhebt, ${ }^{24}$ insgesamt zuverläßiger als die umgearbeitete Büste des Getty Museums, welche aufgrund der weitgehenden Verwendung des laufenden Bohrers in Haar und Bart in der mittel- bis spätantoninischen Zeit entstanden sein dürfte. ${ }^{25}$ Hier sind die Motive linearisiert und vereinfacht, so daß manche Details, vor allem im Nackenhaar, verwischt oder vernachläßigt sind. Nach diesen Ausführungen können wir davon ausgehen, daß die wesentlichen Züge des neuen Kopfes im Benaki-Museum, nämlich das breite, fleischige, von Altersmerkmalen gekennzeichnete Gesicht, der in langen schwungvollen Stähnen auf die Brust herab- 
fallende Bart, sowie der Stirn und Schläfen umrahmende füllige Lockenkranz auf das Original zurückgehen und mit ziemlicher Sorgfalt und Genauigkeit wiedergegeben sind. Was die Schädelkalotte betrifft, so dürfte auch hier die Überlieferung der Replik des Benaki-Museums die bessere sein. Denn die hier vorkommenden kürzeren, wulstigen Locken passen besser zum gesamten Habitus des Kopfes als die dicht anliegenden, flach wiedergegebenen Strähnen, wie sie die beiden anderen Repliken zeigen. Diese sind also wohl eine zu Lasten der Kopisten gehende Vereinfachung, was schon aufgrund ihres unterschiedlichen Verlaufs naheliegt.

Allen Repliken gemeinsam ist die asymmetrische Bildung des Kopfes, dessen rechte Hälfte breiter gebildet und weiter vorgewölbt ist als die linke. Diese asymmetrische Bildung, die in der Vorderansicht der Büste im Vatikan (Abb. 9) sowie in den Rückansichten der Büste in Malibu und des Kopfes im Benaki Museum deutlich wahrnehmbar ist (Abb. 4, 8), hängt wohl mit der Wendung des Kopfes nach links zusammen, ${ }^{26}$ welche somit ein ursprünglicher Zug des Originals sein dürfte.

Die hier vorgelegte Replik kann demnach als Ausgangspunkt für eine erneute Diskussion der Datierung des Originals des Typus Vatikan - Getty - Benaki auf neuer Grundlage dienen. Raeder war bei dem Versuch, eine Vorstellung von dem Original zu gewinnen, verständlicherweise von der Vatikanischen Replik ausgegangen, da die in der Wiedergabe von Haar- und Bartlocken schematisierte und zudem weitgehend überarbeitete Replik Getty sich als unzuverlässig herausstellte. Zwar betrachtete er den ausgeprägt klassizistischen Stil des Kopfes aus der Villa Hadriana zu Recht als Merkmal der Kopistenzeit, ${ }^{27}$ glaubte aber trotzdem, daß er zumindest tendenziell bereits im Original vorhanden war, welches er aus diesem Grund für eine auf die Kunst des 4. Jhs. zurückblickende, 'retrospektive' Porträtschöpfung der späthellenistischen Zeit, genauer gesagt der zweiten Hälfte des 2. Jhs. v. Chr., erklärte. ${ }^{28}$ Die Replik im Benaki-Museum macht indessen klar, daß das Vorbild keine kühle, klassizistische Arbeit, sondern vielmehr eine expressive, physiognomisch sinnvolle Schöpfung in der Art der bärtigen Köpfe alter Männer auf attischen Grabreliefs des 4 . Jhs. v. Chr. ${ }^{29}$ gewesen sein muß. Das legen bereits die schwungvoll bewegten Haarlocken und die welligen Bartsträhnen nahe. Daß der Porträttypus Vatikan - Getty - Benaki ein Werk der spätklassischen
Epoche war, zeigt seine typologische Nähe zu Bildnissen, die übereinstimmend um die Mitte bzw. in die 2. Hälfte des 4. Jhs. datiert werden. Am engsten verwandt ist zweifellos der sog. Krates, ${ }^{30}$ insbesondere die am besten erhaltene Replik des Typus in Neapel (Abb. 11), wobei der bärtige Kopf des Benaki-Museums freier und unruhiger und somit stilistisch fortgeschrittener wirkt, was für eine etwas spätere Entstehung des zugrunde liegenden Originals spricht.

Die Entwicklung dieser Reihe von langbärtigen Köpfen mit nackenlangem, die Ohren bedeckendem Haupthaar und eindeutiger Alterscharakterisierung kann anhand der attischen Grabreliefs einigermaßen gut verfolgt werden. Als frühestes Beispiel, auf dem sämtliche genannten Züge vorkommen, darf die Figur eines sitzenden Mannes auf einem Grabrelief in Dresden gelten, das noch vor die Mitte des 4. Jhs., wahrscheinlich gegen 360 v. Chr., zu datieren ist. ${ }^{31}$ Nur wenige Jahre später, wohl um die Jahrhundertmitte, ist ein Relief in Frankfurt entstanden, auf dem zwei alte Männer mit ähnlich gebildeten Köpfen erscheinen. ${ }^{32} \mathrm{Als}$ nächstes Beispiel ist der eindrucksvolle Kopf des alten Vaters auf dem Ilissos-Relief im Athener Nationalmuseum aus der Zeit um 340 v. Chr. zu nennen. ${ }^{33}$ Über diese Stufe führt ein langbärtiger Kopf in Florenz hinaus (Abb. 12), dessen Züge denen des Vaters des Ilissos-Reliefs im Großen und Ganzen ähnlich sind und der ebenfalls in Profil zu sehen ist. ${ }^{34}$ Bei diesem Kopf ist das Inkarnat weicher modelliert und das Haupthaar differenzierter wiedergegeben, weshalb er wohl in das Jahrzehnt 330-320 v. Chr. gehört. Dieser Kopf bietet einen Anhaltspunkt für die Datierung des Porträts Vatikan - Getty - Benaki aufgrund seiner typologischen und stilistischen Verwandtschaft. Ähnlich gebildet sind vor allem die vorspringende untere Stirnhälfte, die langen, welligen Bartsträhnen sowie der wulstige Haarkranz. Auf derselben Entwicklungsstufe stehen die Köpfe zweier gelagerter bärtiger Männer auf dem sog. Charonrelief im Kerameikos (Abb. 13, 14), die aller Wahrscheinlichkeit nach als Seehändler zu deuten sind. ${ }^{35}$ Auch in diesem Fall sind, trotz der schlecht erhaltenen Oberfläche und der nicht gerade hohen Qualität der Ausführung, die Ähnlichkeiten zu unserem Porträt sowohl in der Haarund Barttracht ${ }^{36}$ als auch in der Alterscharakterisierung deutlich erkennbar. Die weitere Entwicklung dieses allgemeinen Kopftypus wird durch die Gestalt des alten 


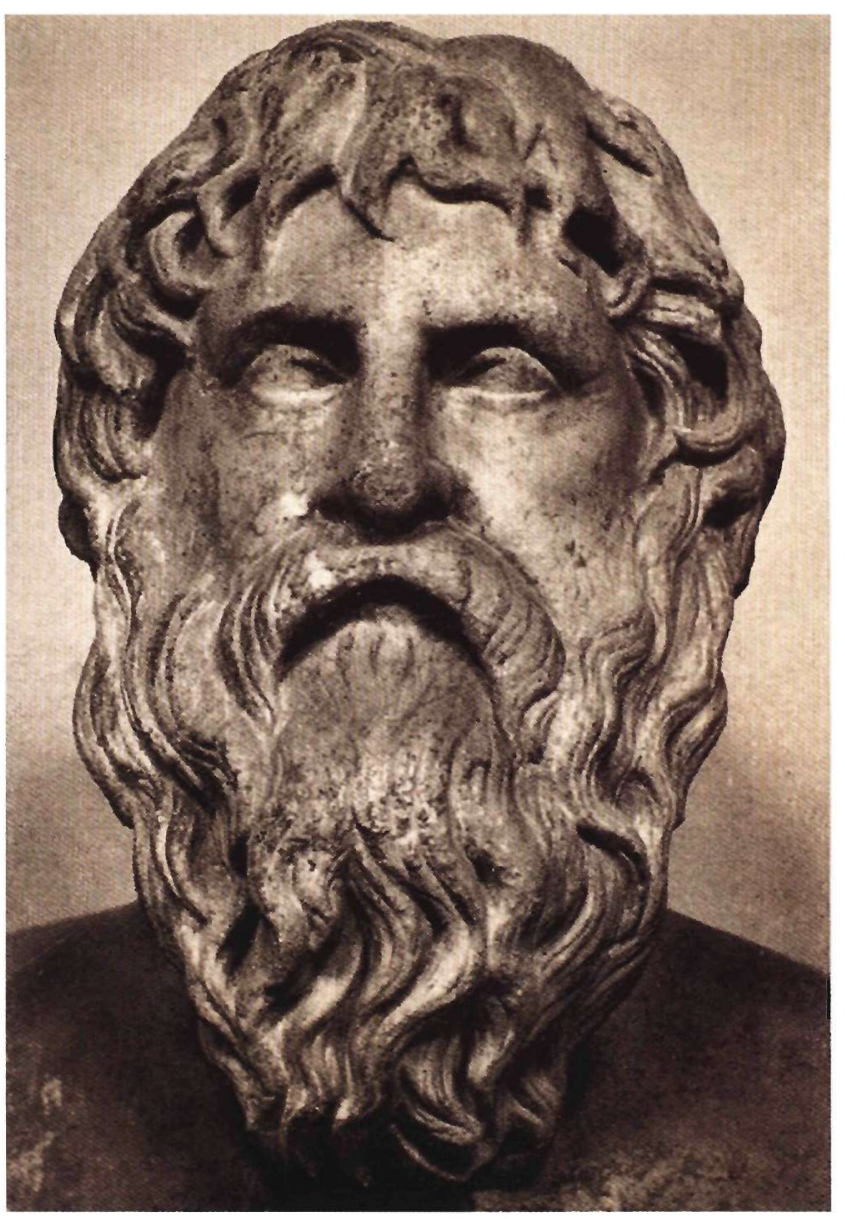

Abb. 11. Bärtiger Marmorkopf. Neapel, Museo Archeologico Nazionale (nach: G. Richter, The Portraits of the Greeks II [London 1965] Abb. 1083).

Hierokles in einem prächtigen Grabnaiskos in Rhamnus aus den Jahren um $320 \mathrm{v}$. Chr. vor Augen geführt ${ }^{37}$-auch wenn der Bart in diesem Fall weniger imposant ist. Hier ist die Modellierung des Inkarnats noch weicher und differenzierter geworden, während die Haar- und Bartsträhnen als wulstigere, durch tiefe Furchen voneinander getrennte Gebilde wiedergegeben sind. Demnach liegt eine Datierung des Originals unseres Porträts gegen 330 bzw. in die 20er Jahre des 4. Jhs. v. Chr. nahe.

Die Frage nach der Identität des Dargestellten kann nur mit Hilfe von allgemeinen Überlegungen und Vermutungen beantwortet werden. Nach Raeder weist die physiognomische Charakterisierung unseres Porträts eindeutig auf einen Philosophen hin: ${ }^{38}$ "Kein Zweifel dürfte darüber aufkommen, daß es sich bei diesem...

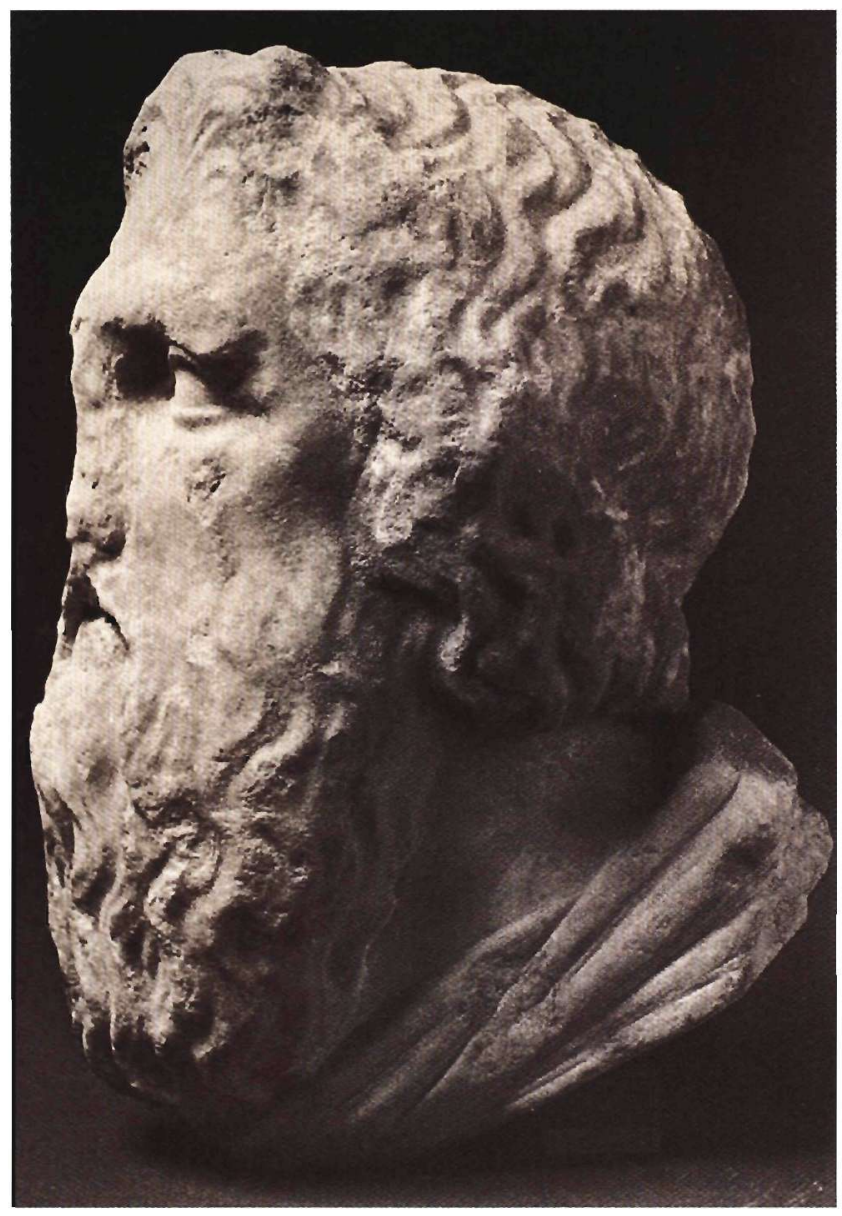

Abb. 12. Kopf eines bärtigen Mannes aus einem attischen Grabrelief. Florenz, Museo Archeologico (Foto: nach Meyer 1989, Taf. 10,4).

Typus um das Bildnis eines Philosophen handelt. Nicht nur der Büstenabschnitt der Replik in Malibu und der mächtige, Würde evozierende Vollbart verraten diesen Rang, auch der hochgewölbte Schädel und die Betonung der steilen Stirn als Sitz des Geistes durch die aufgebauschten Stimhaare charakterisieren den Dargestellten als Denker". Ob die beschriebenen physiognomischen Züge, einschließlich des langen Bartes, auf einen geistig tätigen Mann hindeuten, muß allerdings offen bleiben, ${ }^{39}$ zumal, wie wir gesehen haben, ähnlich gekennzeichnete Figuren auf attischen Grabreliefs "normale" athenische Bürger darstellen, die keineswegs als 'Intellektuelle' zu bezeichnen sind, wie das Beispiel der bereits erwähnten langbärtigen Seehändler auf dem 'Charonrelief zeigt. Andererseits kann die Frisur unseres Porträts 

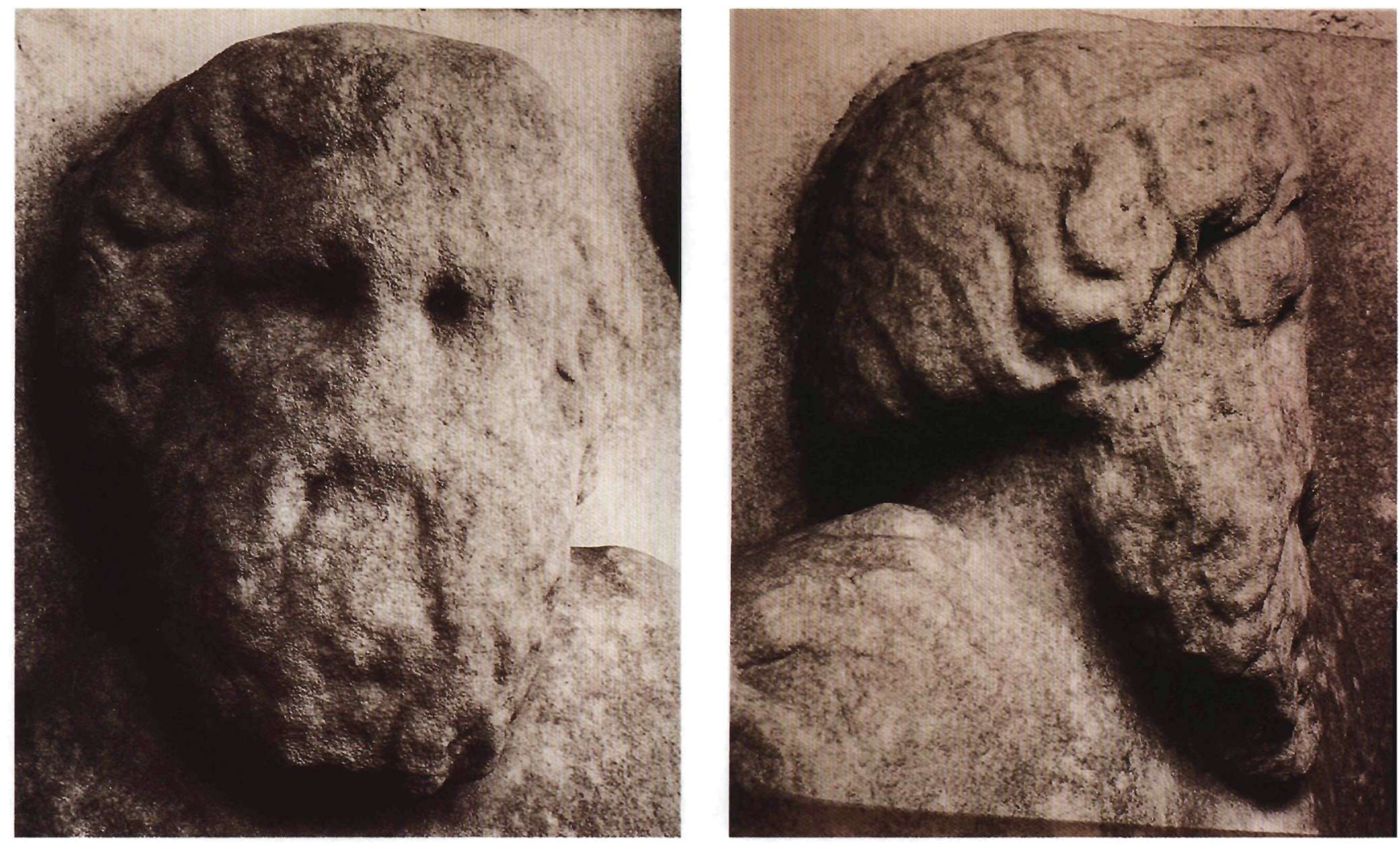

Abb. 13-14. Köpfe bärtiger Männer aus einem Grabrelief im Kerameikos (nach: Bergemann 1997, Taf. 103,1-4).

als Standesabzeichen interpretiert werden. Denn das nackenlange Haar, das einen Lockenkranz bildet, ist wohl als ein Kennzeichen für das gepflegte Aussehen eines Aristokraten anzusehen. ${ }^{40}$ Bezeichnenderweise tritt der aristokratisch gesinnte Oligarch des Theophrast mit mäßig lang geschnittenem Haar auf (Theophr., Char.

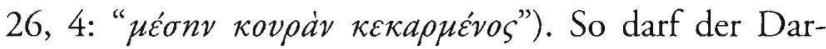
gestellte als ein würdiger Alte gelten.

Ein klares Indiz für die Interpretation der Figur als Philosoph ist dagegen der ohne Chiton getragene Mantel der Replik Getty. Diese seit der archaischen Epoche für Bürgerdarstellungen übliche Bekleidung wurde in der Kaiserzeit für Philosophen geradezu kennzeichnend.41 Nach Dion von Prusa, 72, 2, konnte jeder, der einen

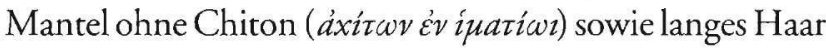
und Bart trug, verspottet, ja sogar angegriffen werden, "obwohl bekannt ist, daß diese Tracht für diejenigen, die Philosophen genannt werden, üblich und gewissermaßen

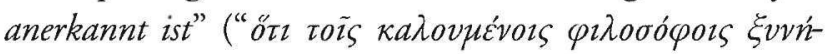

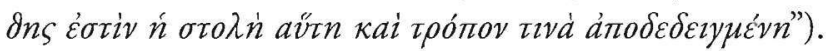

In der Kaiserzeit galt demnach der Dargestellte als ein Philosoph. Daß das Original ähnlich wie die Getty-Büste bekleidet war, ist an sich wahrscheinlich, auch wenn der Mantel möglicherweise anders drapiert war.

Die Herkunft der Büste im Vatikan aus der Villa Hadriana in Tivoli spricht dafür, daß der Dargestellte eine wichtige Persönlichkeit der griechischen Vergangenheit war, die Hadrian besonders hoch schätzte. Leider lassen die nachweislich in der Villa Hadriana gefundenen Kopien griechischer Porträts bisher kein Grundkonzept erkennen, das für ihre Wahl bestimmend gewesen sein könnte. ${ }^{42}$ Andererseits liegt aber aufgrund der zweifellos athenischen Herkunft der neuen Replik im Benaki Museum die Annahme nahe, daß das Original unseres Porträts in Athen stand. ${ }^{43}$ Hadrian war bekanntlich mit den Denkmälern Athens gut vertraut, hatte er doch die Stadt während seiner Regierungszeit dreimal besucht und eine enge Beziehung zu ihr entwickelt. ${ }^{44} \mathrm{Da}$ nun, wie wir gesehen haben, das Original in die Jahre um 330 oder kurz danach gehört, also in die Zeit als der 
traditionsbewußte Politiker Lykurgos die Finanzen Athens verwaltete, liegt der Gedanke nahe, daß es sich bei dem Dargestellten nicht um einen Zeitgenossen, sondern um einen weisen Mann der Vergangenheit handelt. Trifft diese Annahme das Richtige, so erscheint Solon als der wahrscheinlichste Kandidat. Für die Identifizierung als eine besonders ehrwürdige und hochangesehene Persönlichkeit spricht auch die auffällige Größe der Replik aus der Villa Hadriana, die mit einer Gesamthöhe von 64 $\mathrm{cm}$ die beiden anderen, miteinander etwa maßgleichen Kopien bei weitem übertrifft. Das war wohl der Grund, weshalb W. Amelung von einem Götterkopf (Kopf des Poseidon) gesprochen hat, obwohl er die individuellen Gesichtszüge keineswegs verkannte. ${ }^{45}$ Die Größe des Originals wird wohl derjenigen der Kopien Benaki und Getty entsprochen haben, was eine Höhe von etwa $2 \mathrm{~m}$. für eine aufrechtstehende Statue ergibt. Sie war damit ungefähr so groß wie der Lateranische Sophokles, der 2,04 m mißt. Dieselbe Größe kann auch für die nur wenig ältere Platonstatue erschloßen werden. ${ }^{46}$ Auf das von Aischines, Tim. 25, erwähnte, auf der Agora von

\section{NACHTRAG}

Vor kurzem ist ein vollständiger Katalog der antiken Skulpturen im Benaki Museum erschienen (S. Vlizos

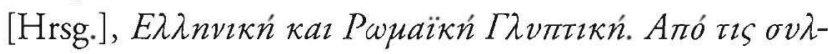

\section{ABKÜRZUNGEN}

Bergemann 1997: J. Bergemann, Demos und Thanatos (München 1997).

von den Hoff 1994: R. von den Hoff, Philosophenporträts des Fruh- und Hochhellenismus (München 1994).

Meyer 1989: M. Meyer, Alte Männer auf attischen Grabdenkmälern, AM 104 (1989) 49-82.
Salamis errichtete Standbild Solons ${ }^{47}$ kann freilich unser Porträttypus schon deshalb nicht zurückgehen, weil dieses nach Zeugnis des Demosthenes, De falsa leg. 251, in die erste Hälfte des 4. Jhs. gehörte. Es gab aber in Athen eine weitere Statue Solons, die im 2. Jh. n. Chr. in der Agora vor der Stoa Poikile stand (Paus., I 16)..$^{48}$ Die Erwähnung dieser Statue in der gegen $325 \mathrm{v}$. Chr. verfaßten zweiten pseudodemosthenischen Rede gegen Aristogeiton (Dem., 26, 23) gibt einen terminus ante quem für ihre Entstehung, der mit der vorgeschlagenen Datierung des hier besprochenen Porträttypus durchaus vereinbar ist. Trotzdem bleibt die Deutung als Solon eine unbestätigte Vermutung und dieser Name sollte höchstens als Spitzname verwendet werden.

Prof. Dr. Emmanuel Voutiras

Department of Archaeology

University of Thessaloniki

54124 Thessaloniki

e-mail:voutiras@hist.auth.gr

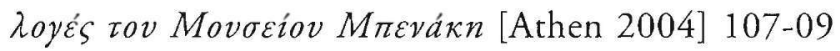
Nr. 25), in dem der hier behandelte Kopf von S. Vlizos vorgelegt wird.
Raeder 1987: J. Raeder, Non traditus vultus. Bildnis eines griechischen Philosophen im Getty Museum, in: Portraits in the J. Paul Getty Museum I (1987) 5-16.

Zanker 1995: P. Zanker, Die Maske des Sokrates. Das Bild des Intellektuellen in der antiken Kunst (München 1995). 


\section{ANMERKUNGEN}

* Für die Anregung zum Studium des hier voregelegten Marmorkopfes sowie für bereitwillige Hilfe bin ich dem Direktor des Benaki Museums Prof. A. Delivorrias und seinen Mitarbeitern Dr. D. Damaskos und Dr. St. Vlizos zu herzlichem Dank verpflichtet. Manchen guten Rat verdanke ich meinem Lehrer G. Despinis. Meine Kollegin B. SchmidtDounas hat freundlicherweise die Mühe auf sich genommen, meinen deutschen Text durchzulesen und zu verbessern; ihr sei auch an dieser Stelle gedankt.

1. Inv. Nr. 39282. Höhe: $40 \mathrm{~cm}$, Breite $26 \mathrm{~cm}$, Tiefe: 27 $\mathrm{cm}$. Die frühere Besitzerin wollte keine näheren Angaben zum Fundort machen; ihre Familie ist aber seit mindestens drei Generationen in Athen ansässig, so daß die Annahme, daß sie in Athen gefunden wurde, naheliegt. Das Material (aller Wahrscheinlichkeit nach pentelischer Marmor) spricht ebenfalls für eine Herkunft aus Athen.

2. Die Herkunftsbestimmung des Marmors (der auch G. Despinis zustimmt) beruht auf empirischen Daten. Eine chemische oder spektrographische Analyse wurde nicht durchgefiihrt.

3. Diesen Hinweis verdanke ich G. Despinis. Die Einwirkung des Feuers auf Marmor führt zur Abspaltung kleinerer oder größerer Splitter, was eine völlige Zerstörung der Oberfläche zur Folge hat; vgl. J. Riederer, The decay and conservation of marbles in archacological monuments, in: N. Herz - M. Waelkens (Hrsg.), Classical Marble: Biochemisry, Technology, Trade (Dordrecht-Boston-London 1988) 472 mit Abb. einer Marmorsäule, die besonders starke Brandschäden zeigt.

4. Zum Einsetzen von Kopien griechischer Porträts auf Hermen s. A. Stähli, Ornamentum Academiae: Kopien griechische Bildnisse in Hermenform, ActaHyp 4 (1992) 147-72, bes. 154-58.

5. Athen, Agora Museum Inv. Nr. S335: E. B. Harrison, Portrait Sculptrure (= The Athenian Agora I [Princeton 1953]) 38-41 Nr. 28 Taf. 19. Harrison weist mit guten Argumenten die zunächst aus typologischen Gründen vorgeschlagene Datierung des Porträts in die Regierungszeit des Commodus oder gar des Septimius Severus zurïck.

6. Die Locken sind bei beiden Köpfen unabhängige, durch Bohrerfurchen getrennte wulstige Strähnen, deren Oberfläche durch feine, dicht angeordnete Ritzlinien gegliedert ist.

7. Über die Zuschreibung kaiserzeilicher Bildnisköpfe an bestimmte Werkstätte und deren methodischen Voraussetzungen s. D. Soechting, Die Porträts des Septimizus Severus (Bonn 1972) 83-106, 275-82; J.-Ch. Balty, Style et facture. Notes sur le portrait impérial romain du IIle siècle de notre ère, RA (1983) 301-15; C. Evers, Les portraits d'Hadrien. Typologie et ateliers (Bruxelles 1994) 295-300. Insbesondere zu den attischen Porträtwerkstätten der Kaiserzeit s. Harrison (Anm. 5) 3; F. C. Albertson, A Bust of Lucius Verus in the Ashmolean Museum, Oxford, and its Artist, AJA 87 (1983) 153-63; K. Fittschen, Eine Werkstatt attischer Porträtbildhauer im 2. Jh. n. Chr., in: Chr. Reusser (Hrsg.), Griechenland in der Kaiserzeit.
Neue Fund und Forschungen zu Skulptur, Architektur und Topographie. Kolloquium zum sechzigsten Geburtstag von Prof. Dietrich Willers, Bern 12-13 Juni 1998 (Bern 2001) 71-78.

8. Derartige Bärte kommen bei kaiserzeitlichen Porträts in der Regel nicht vor. Als Ausnahmen wären das Porträt des Kaisers Pertinax sowie einige Athener Kosmetenporträts, die z.T. auf klassische Vorbilder zurückgreifen, zu nennen; s. E. Lattanzi, I ritratti dei cosmeti nel Museo Nazionale di Atene (Roma 1968) 45-46 Nr. 12, 62 Nr. 30. Zum 'Philosophenbart' als Merkmal des 'Intellektuellen-Zeitgesichts' im späteren 2. Jh. n. Chr. s. Zanker 209-18, dessen Deutung von Hadrians Bart aber fragwürdich ist; s. W. Fischer-Bossert, Der Porträttypus des sog. Plotin. Zur Deutung von Bärten in der römischen Porträrkunst, $A A$ (2001) 149-52. In der klassischen Kunst ist der lange Bart hauptsächlich als Kennzeichen des Alters und der Würde geläufig, was aber andere Konnotationen nicht ausschließt (s. unten Anm. 39).

9. Malibu, J. Paul Getty Museum, Inv. Nr. 71.AA.284: J. Frel, Greek Portraits in the J. Paul Getty Museum (Malibu 1981) 94-95 Nr. 44; Raeder 1987, 8, Abb. 1 a-d.

10. Vatikan, Museo Pio Clementino, Inv. 627: W. Amelung, Die Skulpturen des Vatikanischen Museums 2 (Berlin 1908) 54647 Nr. 356 A Taf. 71; Raeder 1987, 8-10, Abb. 2 a-b.

11. Frel (Anm. 9); Raeder 1987, 8.

12. Amelung (Anm. 10); Raeder 1987, 8-9.

13. Raeder 1987, 8.

14. Raeder 1987, 8 mit Anm. 12.

15. Raeder 1987, 8 Anm. 13. In der frühchristlichen Kunst werden bestimmte Figuren, etwa die Apostel oder die Propheten, im Typus von alten Philosophen dargestellt; $s$. Zanker 1995, 284-88. Zu den Darstellungen später Philosophen vgl. ebenda 288-300.

16. Hier könnte man an eine Reparatur mit Stuck denken.

17. Raeder 1987, 8 mit Anm. 13.

18. Raeder 1987, 9. Amelung (Anm. 10) hielt den Büstenabschnitt für den modern bearbeiteten Rest einer Statue, was Raeder mit Recht bestreitet.

19. Raeder 1987, 9.

20. Raeder 1987, 9.

21. Die kleinen Abweichungen der Vatikanischen Replik in der Bildung der Schläfenlocken sind möglicherweise als Folge der Vergrößerung dieses Kopfes gegenüber dem Original (s. unten) zu erklären.

22. Ein Beispiel dafür sind die bereits oben erwähnten nach hinten gebogenen Batsträhnen auf der linken Seite, die auf dem Hals liegen.

23. Zur Datierung und Würdigung der Büste im Vatikan s. ausführlich Raeder 1987, 9-11. 
24. Raeder 1987, 10.

25. Vgl. Raeder 1987, 10: mittelantoninisch.

26. L. Schneider, Asymmetrie in der griechischen Kunst vom 5. Jh. bis zum Hellenismus (Wiesbaden 1973) 46-49, 66-67.

27. Raeder 1987, 10-11.

28. Raeder 1987, 13-16.

29. Zu diesen Köpfen s. allgemein Meyer 1989; Bergemann 1997, 102-04. Zur Gestaltung ihrer teilweise porträthaft wirkenden Gesichtszüge s. B. Schmaltz, Bildnisse griechischer Grabreliefs, in H. von Steuben (Hrsg.), Antike Porträts. Zum Gedächtnis Helga von Heintze (Möhnesee 1999) 27-33; vgl. Bergemann 1997, 115-16.

30. So bereits Raeder 1987. Zum sog. Krates s. G. M. A. Richter, The Portraits of the Greeks II (London 1965) 185-86 Abb. 1076-78, 1080, 1083; von den Hoff 1994, 19 Anm. 21; 126-27 mit Anm. 68-71; K. Schefold, Die Bildnisse der antiken Dichter, Redner und Denker. Neubearbeitet unter Mitarbeit von A.-C. Bayard - H. A. Cahn - M. Guggisberg - M. T. Jenny - C. Schneider (Basel 1997) 172 Abb. 80, 503; D. Piekarski, Anonyme griechische Porträts des 4. Jhs. v. Chr. Chrnologie und Typologie (Rahden/Westfalen 2004) 96-97, 194 Nr. 32 Taf. 11-13.

31. Dresden, Albertinum, Inv. Nr. ZV2440: H. Diepolder, Die attischen Grabreliefs (Berlin 1931) 43 Taf. 39,1; Bergemann 1997, 169 Nr. 452 Taf. 96, 3.

32. Frankfurt/Main, Liebieghaus, Inv. Nr. 1388: Bergemann 1997, 169 Nr. 457 Taf. 106,1.

33. N. Himmelmann-Wildschütz, Studien zum Ilissos-Relief (Berlin 1954); Bergemann 1997, 165 Nr. 300 Taf. 106,2.

34. Florenz, Museo Archeologico, Inv. Nr. 13780: Meyer 1989, 64 mit Anm. 89 Taf. 10,3-4; Bergemann 1997, 169 Nr. 456.

35. Grundlegend: A. Scholl, Das 'Charonrelief im Kerameikos, JdI 108 (1993) 353-73; s. auch Bergemann 1997, 174 Nr. 598 Taf. 103,1-4.

36. Angesichts dieser engen Verwandtschaft, insbesondere in der Haar- und Barttracht, erscheint Scholls Folgerung a.O. 363, daß es sich bei den Männern des 'Charonreliefs' aus physiognomischen Gründen "um aus dem skythischen Raum stammende Metöken handeln könnte", nicht völlig gesichert.

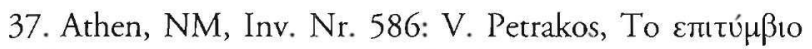

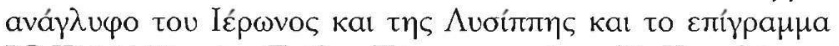

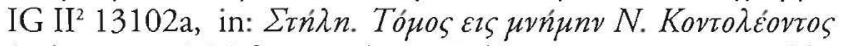
(Athen 1980) Taf. 187; ders., Ephem. 1987, 290-91, Abb.

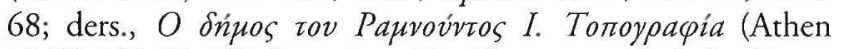
1999) 389-92; W. Geominy, Die Florentiner Niobiden (Bonn 1983) 244-45 Abb. 268-69; Bergemann 1997, 175 Nr. 660; Schmaltz (Anm. 29) 31 Taf. 7,3.

38. Raeder 1987, 11. Zur Problematik der Entstehung des
'Philosophenporträts' als Gattung s. neuerdings von den Hoff 1994, 23-33.

39. Ob der lange Bart "in klassischer Zeit geradezu als Altersmerkmal verstanden werden" soll (so Bergemann 1997, 102; vgl. Meyer 1989, 64), oder ggf. weitere Konnotationen hatte, kann hier nicht diskutiert werden (von den Hoff 1994, 30 mit Anm. 80 und 81 läßt die Frage offen). Es sei nur auf ein Fragment aus der Mittleren Komödie hingewiesen, aus dem hervorgeht, daß der langbärtige Philosoph (von Platons Akademie) bereits im 4. Jh. ein erkennbarer Menschentypus war: PCG V, Ephippos Fr. 14. In späteren Zeiten ist die Assoziation zwischen langem Bart und "Denkerphysiognomie" durchaus geläufig: Plut., Qaest. conv. 710b; Lukian, Pisc. 11 und 37; Philops. 5; Dial. mer. 10, 1; Anonymi Byzantini Physiognomonica 1 (R. Foerster, Scriptores physiognomonici graeci et latini II [Leipzig 1893] 225). Vgl. E. Voutiras,

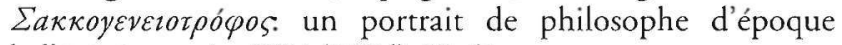
hellénistique, AntK37 (1994) 59-61.

40. Meyer 1989, 176 mit Belegen. Zur 'Nackenhaarfrisur' auf attischen Grabreliefs s. Meyer 1989, 75-78, die zu folgendem Schluß kommt: "Die Nackenhaarfrisur verstehe ich als einen Versuch, Alter zu Veranschaulichen und dabei das Unschöne zu vermeiden". Vgl. Bergemann 1997, 102-03: "Schema mit Anastole und Lockenkranz".

41. Zanker 1995, 216-21; R. R. R. Smith, Cultural Choice and political Identity in Honorific Portrait Statues in the Greek East in the Second Century A.D., JRS 88 (1998) 65 mit Anm. 47; B. C. Ewald, Der Philosoph als Leitbild. Ikonographische Untersuchungen zu römischen Sarkophagreliefs (= RM 34. EH., 1999) 13-16; E. Voutiras, Прото

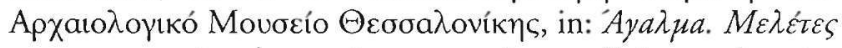

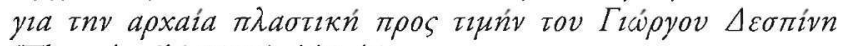
(Thessaloniki 2001) 443-45.

42. $\mathrm{Zu}$ den Kopien griechischer Porträts aus Hadrians Villa in Tivoli s. zuletzt M. G. Picozzi, Un ritratto di Villa Adriana e il busto inv. 525 della Stanza dei Filosofi del Museo Capitolino, in: Studi in memoria di Lucia Guerrini (=Studi Miscellanei 30 [1996]) 133-35.

43. Vgl. G. Lippold, Kopien und Umbildungen griechischer Statuen (München 1923) 44-47.

44. Über die Beziehung Hadrians zu Athen s. D. Willers, Hadrians Panhellenisches Programm (= AntK 16. Beih. [1990]) 7-12.

45. Amelung (Anm. 10).

46. K. Vierneisel, Wie groß war Platons Statue in der Akademie?, in: von Steuben (Anm. 29) 15-26.

47. Richter (Anm. 30) 212-13; Zanker 1995, 52-53, 32425 Anm. 10.

48. Weitere Zeugnisse bei R.E. Wycherley,. Literary and Epigraphical Testimonia (= The Athenian Agora III [Princeton 1957]) 39 Nr. 80; 216 Nr. 709-10. 


\section{EMMANOYHA BOYTYPA}

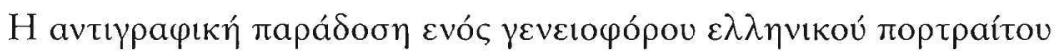

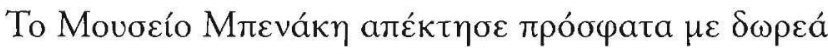

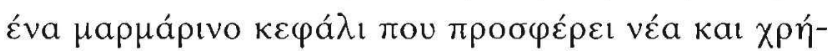

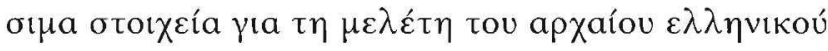

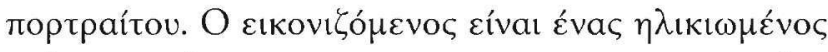

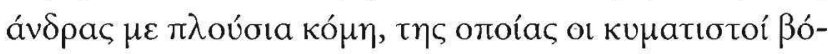

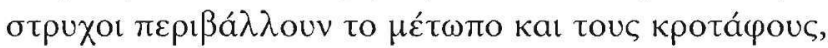

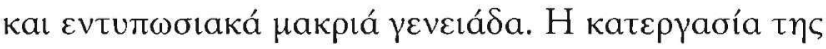

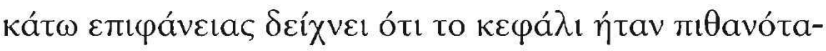

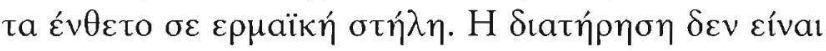

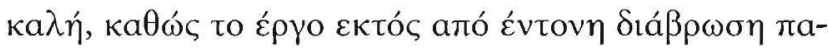

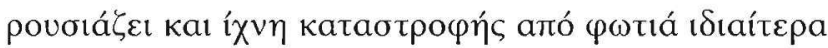

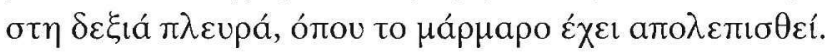

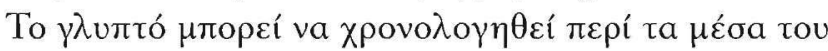

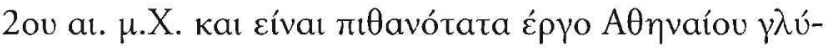

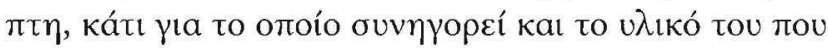

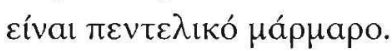

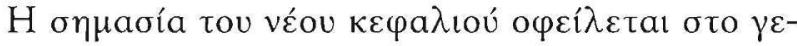

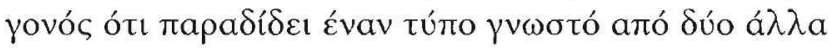

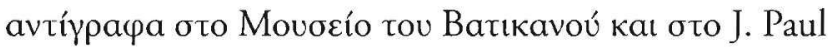

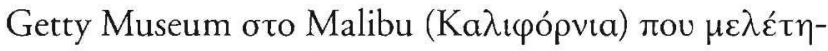

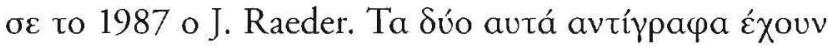

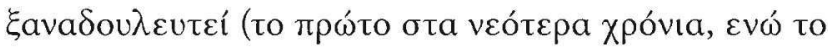

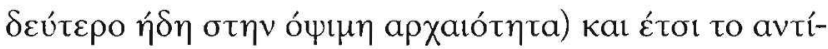

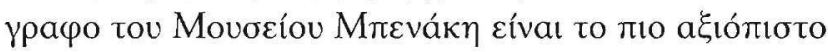

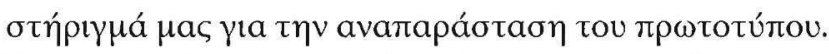

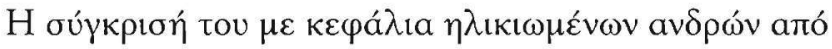

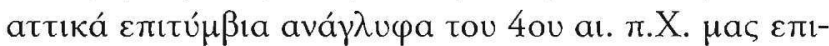

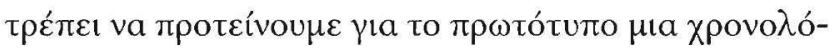

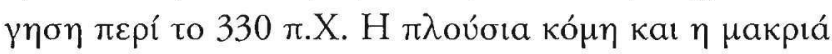

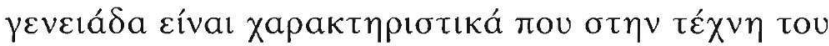

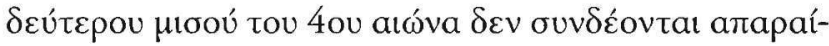

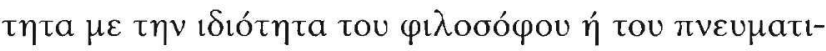

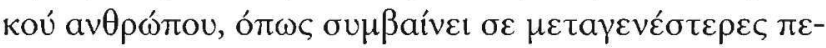

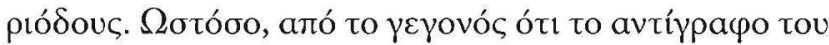

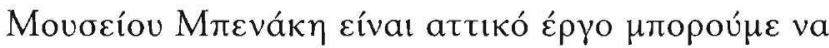

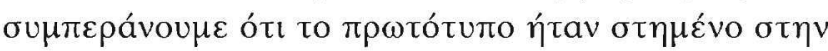

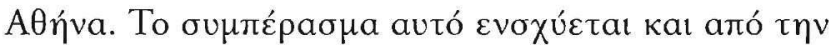

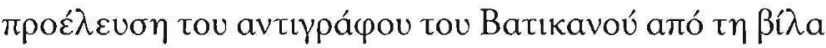

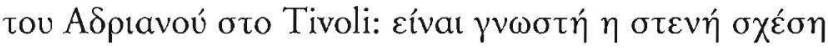

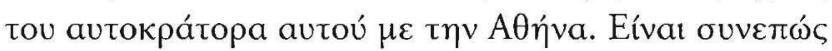

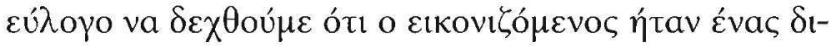

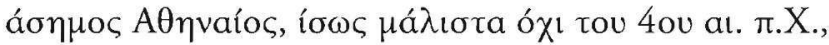

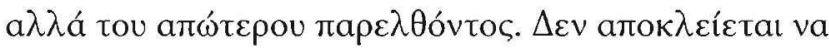

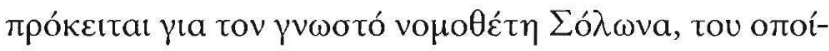

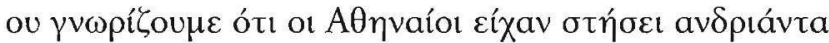

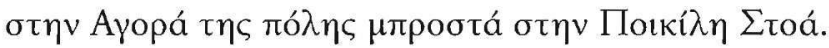

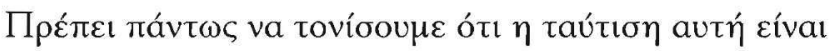

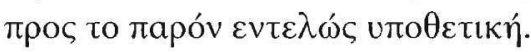

